\title{
Research on InSAR Monitoring Precision Analysis of Coal Mining Influence Based on Long Time Sequence
}

\author{
Junhai Gao ${ }^{1,2}$, Rongjiu $\mathrm{Gao}^{2}$, Lisheng Chen², Yongbin $\mathrm{Han}^{2}$, Jiancheng Yan ${ }^{1,2, *}$ and Xingli $\mathrm{Li}^{2}$ \\ ${ }^{1}$ Tangshan Branch, China Coal Science and Technology Ecological Environment Technology Co, Ltd, ,Tangshan 063012,China; \\ ${ }^{2}$ Tiandi(Tangshan) Mining Science and Technology Company Ltd.,Tangshan 063000,China
}

\begin{abstract}
In this paper, in view of the scope of coal mining subsidence, the magnitude of subsidence deformation and the impact on buildings (structures), InSAR technology is selected for monitoring, time series analysis method combined with D-InSAR method for processing, and Sentinel- 1 Satellite IW data and RADARSAT-2 satellite XF data have been monitored for four years in the mining-affected area and its surrounding areas. The results show that there are obvious subsidence ranges on the surface of the current mining area and the unstable settlement area. The Shen District has been in a stable state in recent years, and the time sequence analysis for more than 3 years has verified the high accuracy and reliability of the monitoring results. The analysis of the monitoring results shows that the InSAR technology can be used for mining subsidence monitoring, especially when determining the subsidence boundary and whether it is stable or not, it has its technical superiority.
\end{abstract}

\section{Introduction}

The surface of coal mining produces subsidence and deformation. The scope, degree of influence, development trend and deformation law of mining subsidence are the current research hotspots in the industry. The research methods mainly include the establishment of observation stations, comprehensive analysis and computer numerical simulation[1], Laboratory simulation of similar materials, etc. In recent years, the application of technology such as total station robots, laser scanners [2,3], and drones [4,5], especially the development and application of technologies such as remote sensing and InSAR [6-10], it provides a new method for obtaining three-dimensional data information of mining subsidence. The advantages of InSAR technology applied to deformation monitoring are as follows: the analysis of the monitoring dimension and deformation law of traditional monitoring methods stays at the "point line" level, which can not realize regional monitoring, while InSAR can improve the mining surface deformation monitoring from the "point line" level to the "area" level.Traditional monitoring methods can not ensure the acquisition of deformation information in the key deformation period, while InSAR can extract the deformation information of the previous surface affected by mining using historical data, so as to trace the past deformation; the surface deformation characteristics obtained by traditional monitoring methods are not really "the same state". A monitoring method that can instantly obtain the surface state information of the entire area of interest is needed. InSAR can quickly scan the entire monitoring area through satellites, and achieve simultaneous acquisition of the monitoring target area. result.
This paper applies InSAR technology and adopts time series processing methods to monitor the building group and its surrounding area at the boundary of the mining-affected area, and analyze in detail to determine whether the building group is located in the subsidence deformation area and the degree of influence, and solve the local government and villagers, concerns with scientific methods Security issues. The key monitoring area this time is about 30 $\mathrm{km}^{2}$. While implementing InSAR monitoring, leveling of the monitoring area was carried out, which can be compared and analyzed to verify the accuracy and reliability of the results.

\section{Analysis and Selection of Interferometric Methods}

According to the analysis of monitoring purposes and site conditions, the D-InSAR differential interferometry monitoring and analysis method is selected to carry out coherent target timing analysis, which is convenient for grasping the impact of long-term coal mining and determining the impact range and degree changes in recent years.

D-InSAR interferometric measurement method: Synthetic Aperture Radar Differential InSAR (D-InSAR) technology refers to the use of two interferometric images in the same area, one of which is the interferometric phase image before deformation, and the other is after deformation The acquired interferometric phase image is then processed by differential processing (removing the influence of the earth's curved surface and topographical undulations) to obtain the measurement

${ }^{*}$ Corresponding author: jc_yan@163.com 
technology of surface deformation.

With the help of the collected high-precision DEM in the monitoring area, the 2-pass differential interference is selected this time. The principle of the two-pass method is as follows: It is assumed that there is a deformation in the SAR observation slope direction during the time period when the two SAR images are acquired. The interference phase $\phi_{1}$ formed by the interference of two SAR images can be expressed as:

$$
\begin{gathered}
\phi_{1}=-\frac{4 \pi}{\lambda}\left[\left(R_{1}+\Delta r\right)-R_{2}\right] \\
\phi_{0}=-\frac{4 \pi}{\lambda}\left[R_{1}-R_{2}\right] \\
\Delta \phi=\phi_{1}-\phi_{0}=-\frac{4 \pi}{\lambda} \Delta r
\end{gathered}
$$

$R_{1}$ and $R_{2}$ are respectively the slant distance of the main and auxiliary images, $\lambda$ is the wavelength, and $\phi$ is the phase simulated by the existing DEM according to the interference baseline and incident angle. The differential interference result is only related to the wavelength, not to the baseline

Coherent target timing analysis method: The project uses the coherent target timing analysis combined with Small Baseline Subset (SBAS) and Persistent Scatterer InSAR (PS-InSAR) technology to combine all SAR images covering the same area. Several sets are formed, and the coherent target points are calculated based on the small baseline set combination principle. The principle is: the SAR image interference baseline distance (including time baseline distance and spatial baseline distance) in the collection is small, and the baseline distance between subsets is large. Then after simple and effective merging, all available small baseline interferograms are obtained. The merging here is based on the minimum deformation rate standard, which is easily obtained by using the singular value decomposition (SVD) method. The SBAS-InSAR method limits the geometric decoherence caused by the long baseline, and makes more SAR images participate in the deformation calculation, increasing the time sampling.

Assuming that there are $\mathrm{M}(\mathrm{M}>2)$ scene $\mathrm{SAR}$ images, according to the imaging time sequence, set the cumulative shape of the same resolution unit $(x, r)$ at imaging time $\mathrm{i}(\mathrm{i}=0,1, \ldots, M-1)$ The variable phase is $\phi\left(t_{i}, x, r\right)$. If the critical baseline condition is met, there are $N$ different interference image pairs, which can generate $\mathrm{N}$ scene differential interferograms:

$$
\frac{M}{2} \leq N \leq \frac{M(M-1)}{2}
$$

\section{Data selection and processing}

\subsection{Data selection and collection}

The research work collected necessary InSAR data, DEM data and basic geographic information data for cartographic analysis.

\subsubsection{Overview of the project research area}

The research project area is located in the Kailuan mining area, and the southwest of the monitoring area is the mining area. The location of the project area is shown in Figure 1.

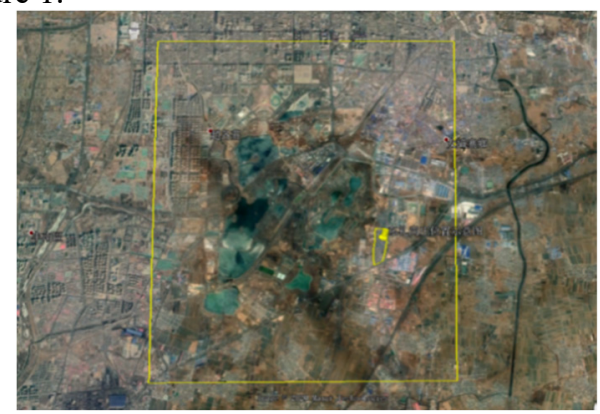

Figure 1 Schematic diagram of the monitoring area

\subsubsection{Radar data}

Sentinel-1 satellite IW mode radar data and RADARSAT-2 satellite XF mode radar data are used this time, of which RADARSAT-2 satellite XF mode radar data is the main data source. The Sentinel-1 satellite has a replay cycle of 12 days, C-band, and IW data in the interference wide mode is selected. The single-scene data coverage is $250 \mathrm{~km} \times 180 \mathrm{~km}$. The data cycle is from April 2017 to November 2018, with 48 periods. The RADARSAT- 2 satellite has a return period of 24 days, C-band, and XF data in scan mode. The single-scene data coverage is $125 \mathrm{~km} \times 125 \mathrm{~km}$. The data period is from November 2016 to December 2019 , with a total of 34 periods.

\subsubsection{SRTM DEM data in the work area}

The horizontal resolution is 90 meters ( 3 seconds), the elevation accuracy of the monitoring area is about 10 meters, and the terrain of the work area is flat, which meets the requirements of terrain compensation in InSAR processing

\subsection{Data processing analysis}

\subsubsection{Radar data registration}

The registration of SAR image is to accurately fit two or more images of the same target area together in space. Select one scene from the Sentinel-1 sequence radar data and the RADARSAT-2 sequence radar data as the main image (for example, RADARSAT-2 selects the radar imaging time of 20170515 as the main image), and register the remaining scene images as the secondary images. Correct the secondary image to the spatial position of the main image. Orthogonal 
correlation technology is used to achieve precise registration of radar data. The registration accuracy of Sentinel-1 data must be higher than 0.002 pixels, and the registration accuracy of RADARSAT-2 data must be higher than 0.2 pixels.

According to Sentinel-1/RADARSAT-2 main image registration DEM data, through DEM data information and radar imaging geometric information, remove the terrain phase in the subsequent interferometry. Apply the SRTM DEM data covering the test area to transform the coordinate system of the external DEM based on the imaging geometry of the SAR image, that is, geocoding, establish a conversion lookup table between the coordinate system between the SAR image and the external DEM, and simulate the SAR image with the DEM. The texture matching of SAR images has a matching accuracy higher than 0.2 pixels.

\subsubsection{Selection of interference image pair and $D$-InSAR processing}

According to the baseline distribution characteristics of the acquired SAR data sequence, combined with the basic characteristics of regional ground deformation, select the data with a spatial baseline less than $300 \mathrm{~m}$ and a time interval less than 360 days to form an interferometric image pair sequence; interferometric image pairs that meet the baseline requirements are subjected to interference processing. Generate interferogram sequence; introduce precise orbital parameters for baseline estimation, remove flat ground based on geometric imaging model, use DEM sampled into radar coordinate system, simulate terrain phase through geometric imaging model, and obtain the phases including deformation phase, atmospheric phase and noise phase Differential interferogram: Screen the differential interferogram, and eliminate the interferogram with obvious influence of strong convection and the interferogram with obvious residual interference phase fringes.

\subsubsection{Timing analysis of coherent targets}

Perform time series analysis on the interference phase of coherent point targets, estimate atmospheric fluctuations, DEM errors, and noise according to the temporal and spatial characteristics of each phase component, separate them from the differential interference phase one by one, and finally obtain the deformation rate of each coherent point target .

Selection of coherent point targets: Coherent targets are radar targets that have stable backscatter characteristics in time series and maintain a stable phase. These targets are generally point targets or continuous targets. The solution of the surface deformation variables is based on the phase changes of these targets. Generally, to obtain correct statistical results, more than 20 scenes of SAR data are required. The point target detection algorithm considers a point target to be a coherent target, and determines whether it is a relevant target according to the degree of correlation between the sub-views.

Time series differential phase analysis: use the differential interference phase obtained in the previous period to extract the interference phase of the coherent point target, and use the spatial phase unwrapping for the point target phase, construct the Delaunay triangulation network according to the position of these points, and determine the residual in the triangulation network For the handicap, the minimum cost flow algorithm is used to connect the positive and negative residual point pairs, and the unwrapping result of each point is obtained. For the differential interference phase sequence of the coherent point target, the two-dimensional periodogram is used for parameter inversion to estimate the movement rate of adjacent points and the elevation error correction. Through multiple error correction iterations, the correlation coefficient of the two-dimensional periodic function is $0.8-0.85$, accurately extract the linear deformation rate.

\section{InSAR Monitoring Results and Analysis}

Through two processing methods of conventional D-InSAR and coherent target timing analysis, the RADARSAT-2 radar data is used to obtain the average land subsidence rate of the Nanhu area from November 2016 to December 2019 and the cumulative land surface of the three years Subsidence, to obtain the distribution of land subsidence and the development trend of subsidence; use Sentinel-1 radar data to obtain the average deformation rate, cumulative subsidence and subsidence contour map of the Nanhu area from April 2017 to November 2018

\subsection{Monitoring results and analysis of land subsidence in Nanhu area of Tangshan City}

\subsubsection{D-InSAR results based on RADARSAT-2 data}

For the Nanhu area of Tangshan City, we used RADARSAT-2 data to perform multiple phases of interference processing from November 2016 to February 2019, and obtained 6 phases of interference patterns, as shown in Figure 4-1 6. In the figure, a color circle represents a $28 \mathrm{~mm}$ deformation. 


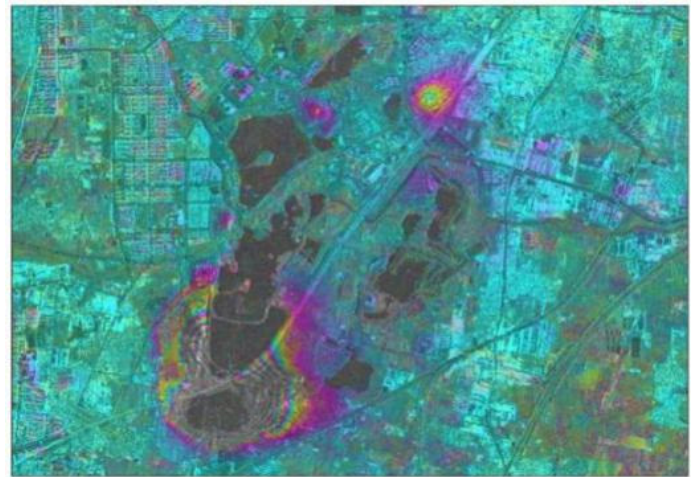

Figure 4-1 Deformation interference pattern of Nanhu area (20161128 20170304)

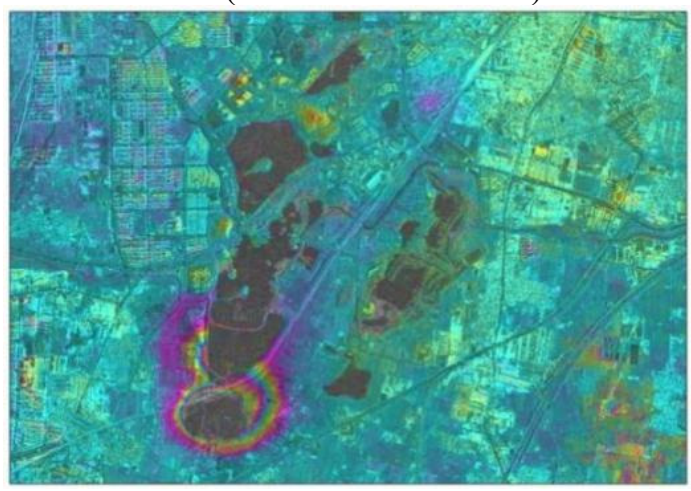

Figure 4-2 Deformation interference pattern of Nanhu area (20161222 20170115)

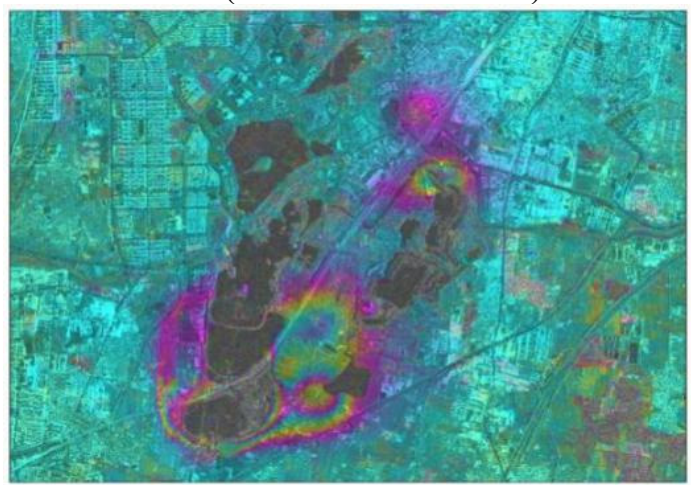

Figure 4-3 Deformation interference pattern of Nanhu area (20171123 20180227)

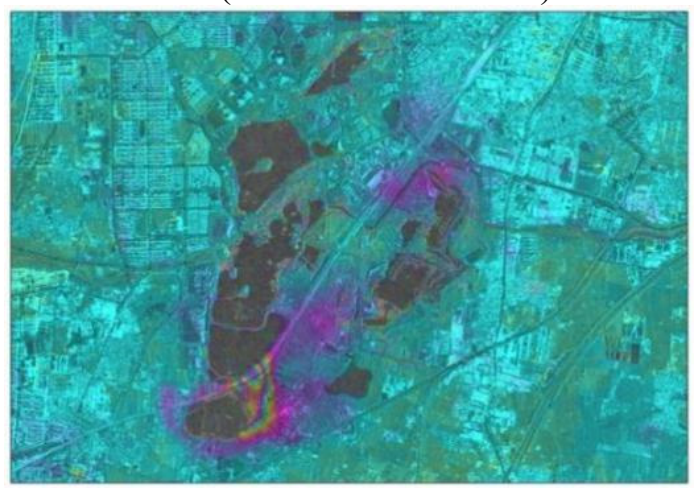

Figure 4-4 Deformation interference pattern of Nanhu area (20180203 20180227)

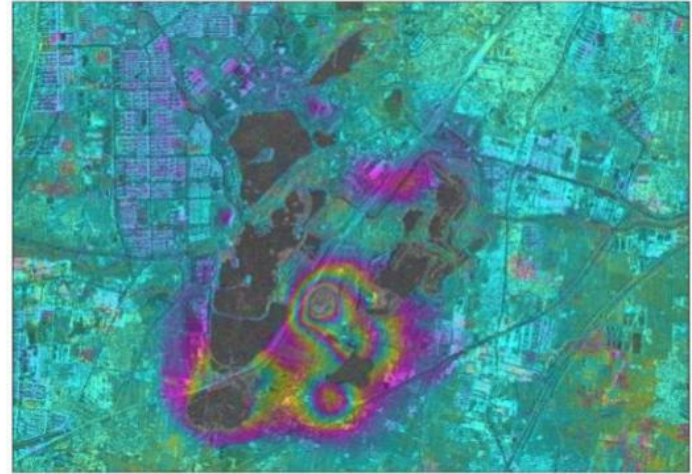

Figure 4-5 Deformation interference pattern of Nanhu area (20181212 20190222)

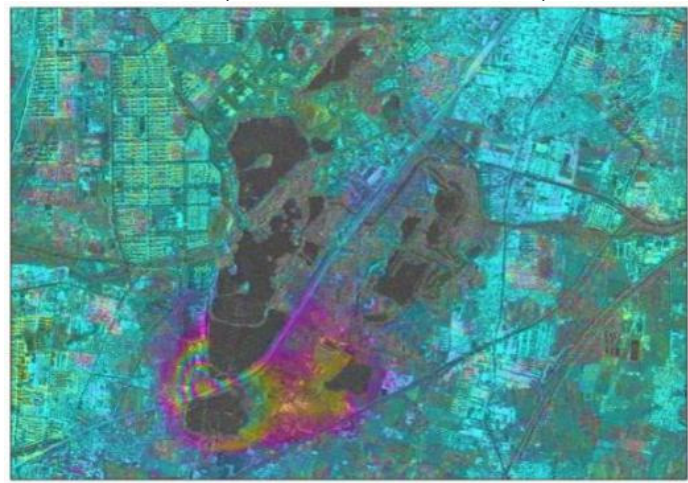

Figure 4-6 Deformation interference pattern of Nanhu area (20191020_20191207)

As can be seen from Figure 4-2, from December 22, 2016 to January 15, 2017, the ground subsidence rate around the mining center in the Yuexu District of Tangshan Mine was rapid, and the deformation center interference fringes exceeded 6 in only 24 days. Color week, both the settlement amount exceeds $160 \mathrm{~mm}$. Other places are uniform in color, and the ground is stable.

It can be seen from Figure 4-4 and Figure 4-5 that in different time periods, with the change of mining activities, the settlement center, settlement range and settlement speed will change accordingly, reflecting the changes induced by coal mining during the monitoring period. Deformation development status.

\subsubsection{Timing analysis results of coherent targets based on Sentinel-1 data}

For the Nanhu area of Tangshan City, using the coherent target time series analysis method and using Sentinel-1 data to obtain the InSAR monitoring results of the settlement of the Nanhu area of Tangshan City (centered on the area affected by mining in Tangshan) from April 2017 to November 2018 The sequence has 5 periods, see Figure 4-7 11. 


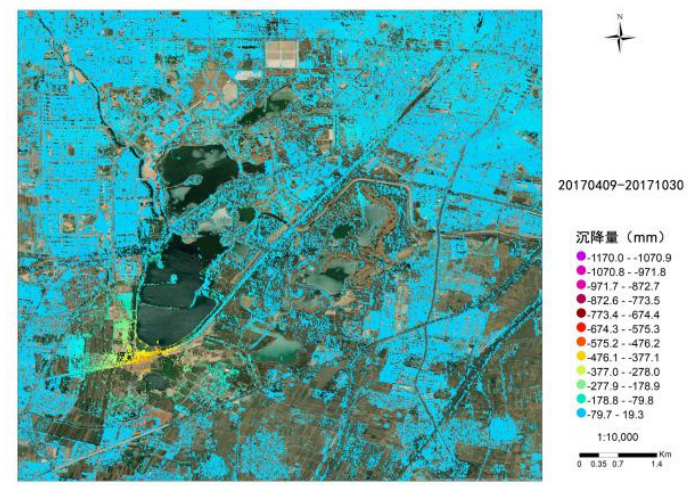

Figure 4-7 InSAR monitoring results of land subsidence in the study area (20170409 20171030)
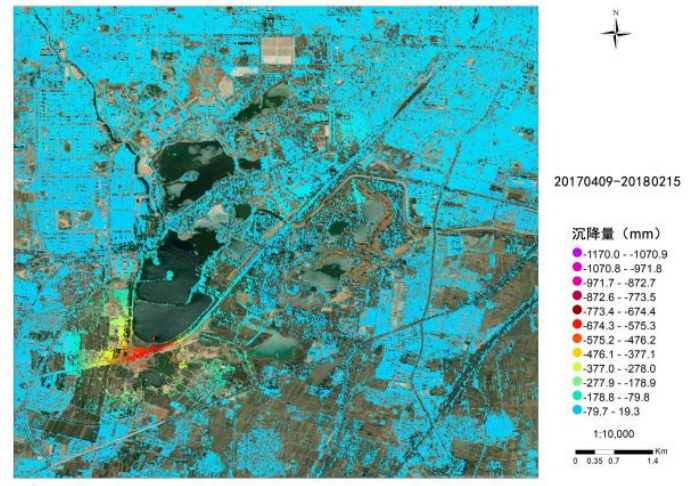

Figure 4-8 InSAR monitoring results of land subsidence in the study area (20170409 20180215)
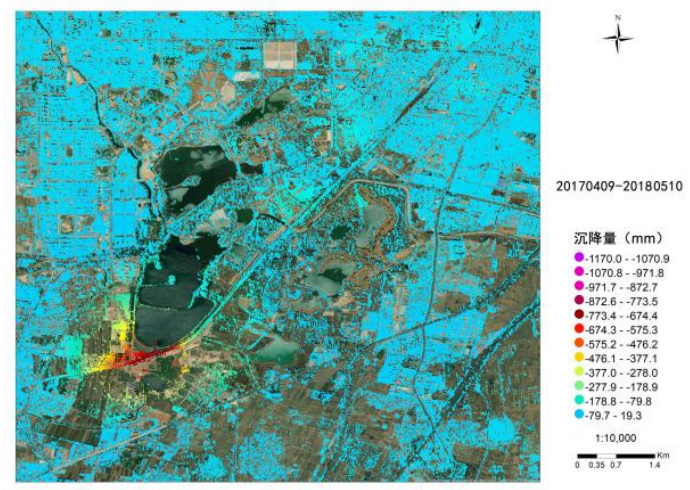

Figure 4-9 InSAR monitoring results of land subsidence in the study area (20170409-20180510)

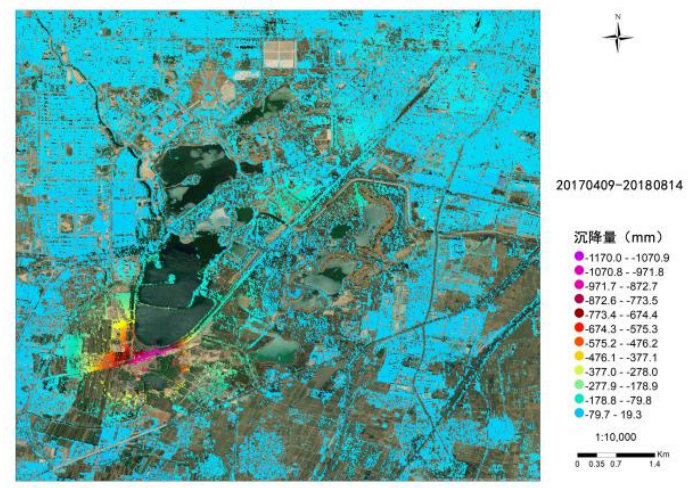

Figure 4-10 InSAR monitoring results of land subsidence in the study area (20170409 20180814)

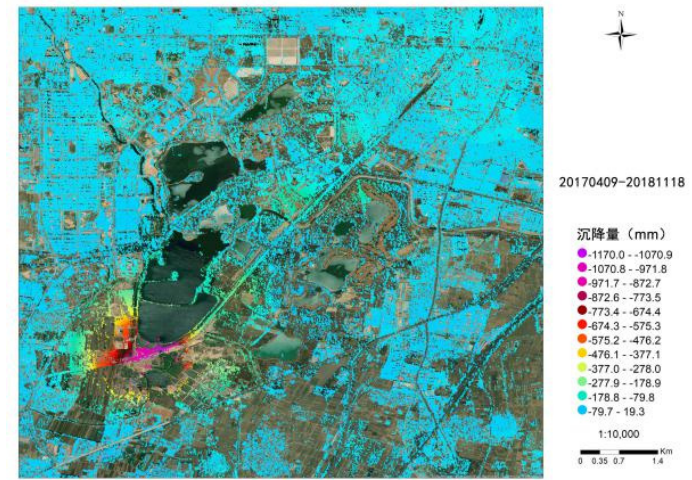

Figure 4-11 InSAR monitoring results of ground subsidence in the study area (20170409 20181118)

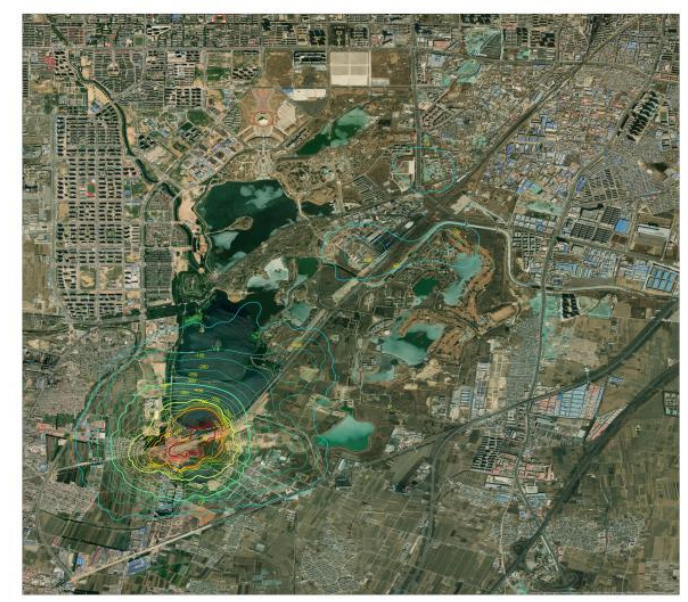

Figure 4-12 Contour map of the subsidence in the study area (20170409 20181118)

It can be clearly seen from the Sentinel-1 monitoring results that the changes in the settlement of the Nanhu area mainly occurred in the southwestern part of the Nanhu Lake, which was the main mining area of coal mines from 2016 to 2018. The monitoring results can also accurately reflect the intensity and scope of underground mining. During this monitoring period, the maximum settlement in this area reached more than $1100 \mathrm{~mm}$. Based on the Sentinel-1 settlement monitoring results, the settlement contour map of the Nanhu area from April 2017 to November 2018 was drawn, as shown in Figure 4-12. It can be clearly seen from the figure that the eastern part of the Nanhu area is generally stable and there is no significant land subsidence.

\subsection{Time series analysis of coherent targets based on RADARSAT-2 in land subsidence in the eastern part of Nanhu Lake}

Based on the 34 phases of RADARSAT-2 satellite XF mode radar data from November 2016 to December 2019, and using the coherent target timing analysis method, the ground subsidence rate map of the eastern region of the monitoring area from November 2016 to December 2019 was obtained, As shown in Figure 4-13. It can be seen from the figure that the impact of land subsidence basically disappears to the east side of Nanhu Lake, and the maximum subsidence rate in the 
figure reaches $28 \mathrm{~mm} / \mathrm{year}$, which is located in the southwest corner of Figure 4-13; the location of the monitoring point marked in the figure (RP Location) The surrounding area is outside the scope of influence of coal mining, and the area is generally stable without significant ground subsidence.

The time series of land subsidence at typical coherent target points (RP points) are analyzed. Figure 4-14 is a time series diagram of land subsidence at a typical target point. It can be concluded from the figure that there is no significant seasonal land subsidence at this point, showing the characteristics of ground stability.

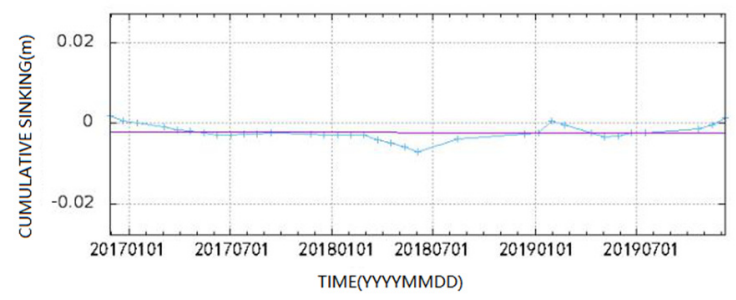

4-13 Time series diagram of land subsidence at typical coherent target points

At the same time, the cumulative subsidence map for the three years starting from November 2016 was obtained. The overall land subsidence development trend in the eastern part of Nanhu Lake is shown in Figure 4-15 17.

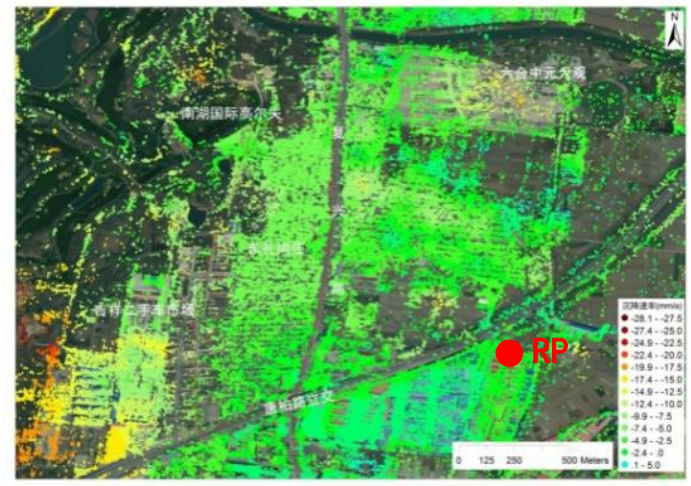

Figure 4-14 Map of land subsidence rate in the eastern part of Nanhu Lake (2016.11 2019.12)

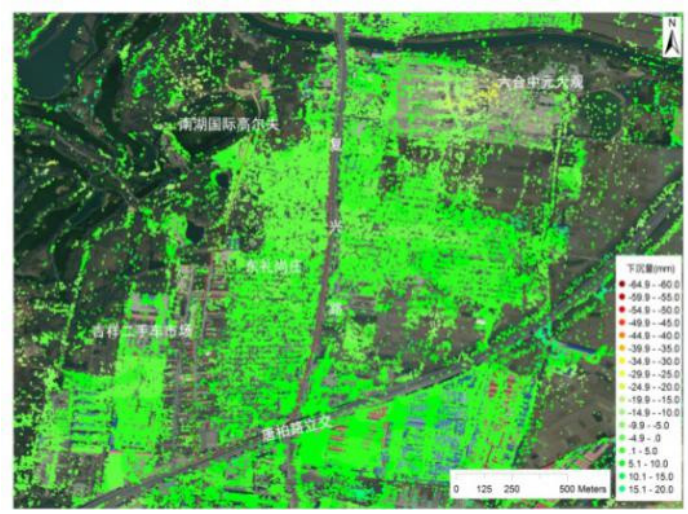

Figure 4-15 Map of cumulative land subsidence in the eastern part of Nanhu Lake (2016.11 2017.11)

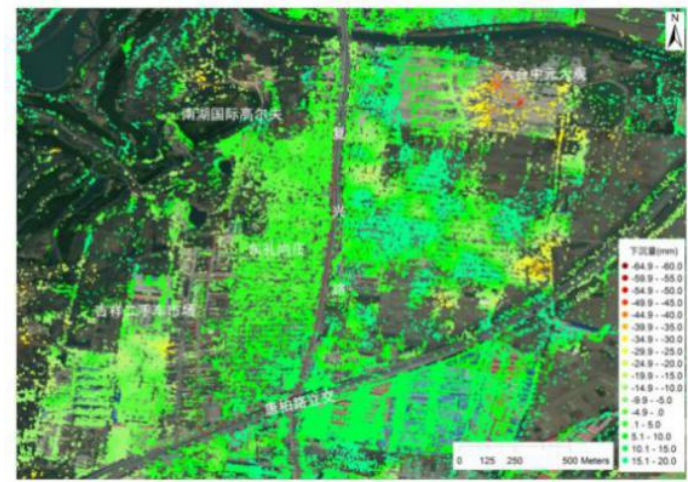

Figure 4-16 Map of cumulative land subsidence in the eastern part of Nanhu Lake (2016.11 2018.12)

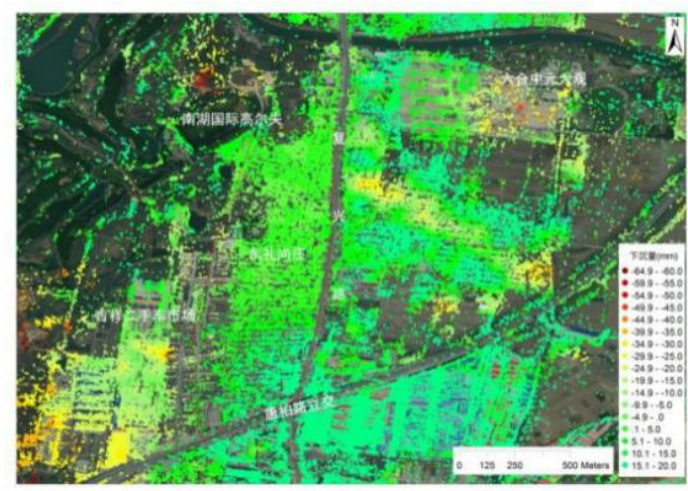

Figure 4-17 Map of cumulative land subsidence in the

eastern part of Nanhu Lake (2016.11 2019.12)

The time series of land subsidence at typical coherent target points and the cumulative land subsidence map show that the eastern area of Nanhu Lake is in a relatively stable condition, with no significant accumulation of land subsidence and no significant uneven settlement.

\section{Conclusion}

Based on the space-borne InSAR technology, the land subsidence monitoring of the Nanhu area from 2016 to 2019 was completed, and information such as the cumulative amount of land subsidence and the average annual subsidence rate in the monitoring area were extracted. Through the long time sequence analysis of the monitoring results for more than 3 years, the high accuracy and reliability of the monitoring results are verified, which can be used for large-scale regional monitoring of coal mining areas.

Through the analysis of the ground subsidence monitoring results, it is concluded that the changes mainly occur in the current mining affected area, and the eastern area of Nanhu Lake has become stable and has stabilized, and there is no significant uneven subsidence.

InSAR technology can be used for mining subsidence monitoring, especially when determining the boundary of subsidence and whether it is stable or not, it has its technological advantages.

For the current mining area of the mining area, Sentinel-1 data increases the time sampling rate and 
reduces the impact of time incoherence, which can enhance the description of the deformation process of the mining center of the mining area. For the slower ground subsidence, the RADARSAT-2 satellite can Describe the details of the deformation field more finely in space. For large deformation areas, that is, InSAR decoherence areas, it can also be supplemented with conventional measurements to improve the current deficiencies of InSAR technology.

\section{Acknowledgement}

This paper is supported by General Project of Science and Technology Innovation and Entrepreneurship Fund of Tiandi Science and Technology Co., Ltd (2018-TD-MS052),National Key R\&D Program of China(2016YFC0501105) and Hebei Province"333 Talent Project " (A202001096).

\section{Referneces}

1. X.Yuan,W.Lyu,D.Yuanet al.Application of Numerical Fitting in Mine Subsidence Monitoring. MINE SURVEYING. 47,6-9(2019).

2. J. Bai, C. Li. Application of Three -Dimensional Laser Scanning Technology in Mines.MINE SURVEYING. 47, 116-119 (2019).

3. C.Guo, J. Li ,H. Feng,et al.The Application of 3D Laser Scanning in Dam Subsidence Monitoring in Mining Area.MINE SURVEYING. 42, 70-72(2014).

4. E.Hou, J. Zhang, X. Xie, et al.Contrast Application of Unmanned Aerial Vehicle Remote Sensing and Satellite Remote Rensing Technology Relating to Ground Surface Cracks Recognition in Coal Mining Area.Geological Bulletin of China. 443-448, 38(2019).

5. F.Tang, L.Li, X.Li, et al. Research on Characteristice of Minging-induced Surface Cracks Based on UAV images.Coal Science and Technology.48, 130-136(2020).

6. F.Tang, L.Dong, Z.Wang, et al.A 3-D inversion model for InSAR detected displacements based on ground subsidence symmetry induced by horizontal coal mining.Journal of China Coal Society.44,210-220(2019).

7. Y. Luan, Y. Liang, Z.Ji, et al.Monitoring and Analysis of Surface Subsidence Caused by SBAS-InSAR Technology. Coal Science and Technology.14,198-204 (2020).

8. T. Liang .Monitoring Surface Deformation in Mining Area Using Short Baseline Set InSAR Technology. Bull Surv Map.S1,82-84 (2014).

9. D. Li, Z. Deng, X. Gao,et al.Monitoring and Analysis of Surface Subsidence in Mining Area Based on SBAS-InSAR. Geomatics and Information Science of Wuhan University.43, 1531-1537(2018).

10. S. Han, B.Zhao, Y.Bai,el al. Mining Subsidence Research Based on Sbas-insar in Yaojie Caol Mine.MINE SURVEYING. 47, 1-5(2019). 